\title{
Super-paramagnetic clustering of yeast gene expression profiles
}

\author{
G. Getz ${ }^{a}$, E. Levine ${ }^{a}$, E. Domany ${ }^{a}$ and M. Q. Zhang ${ }^{b}$ \\ ${ }^{a}$ Department of Physics of Complex Systems, Weizmann Institute of Science, \\ Rehovot 76100, Israel \\ ${ }^{b}$ Cold Spring Harbor Laboratory, P. O. Box 100, Cold Spring Harbor, New York \\ 11724, USA
}

\begin{abstract}
High-density DNA arrays, used to monitor gene expression at a genomic scale, have produced vast amounts of information which require the development of efficient computational methods to analyze them. The important first step is to extract the fundamental patterns of gene expression inherent in the data. This paper describes the application of a novel clustering algorithm, Super-Paramagnetic Clustering (SPC) to analysis of gene expression profiles that were generated recently during a study of the yeast cell cycle. SPC was used to organize genes into biologically relevant clusters that are suggestive for their co-regulation. Some of the advantages of SPC are its robustness against noise and initialization, a clear signature of cluster formation and splitting, and an unsupervised self-organized determination of the number of clusters at each resolution. Our analysis revealed interesting correlated behavior of several groups of genes which has not been previously identified.
\end{abstract}

\section{Introduction}

DNA microarray technologies have made it straightforward to monitor simultaneously the expression levels of thousands of genes during various cellular processes [1][2]. The new challenge is to make sense of such massive expression data [3]. In most such experiments, investigators compare the relative change of gene expression levels between two samples (one is called the target, such as a disease sample; the other is called the control, such as a normal sample). In a typical experiment simultaneous expression levels of thousands of genes are viewed over a few tens of time-points (or different tissues [4]). Hence one needs to analyse arrays that contain $10^{5}-10^{6}$ measurements.

The aims of such analysis are typically to (a) group genes with correlated expression profiles; (b) Focus on those groups which seem to participate in 
some biological process; (c) Provide a biological interpretation of the clusters. Interpretations could be co-regulation of the mean cluster expression with a known process, a promoter common to most of the genes in the cluster, etc. (d) In experiments that compare data from different tissues (such as tumor and normal [4]) one also tries to differentiate them on the basis of their genetic expression profiles.

The sizes of the datasets and their complexity call for multi-variant clustering techniques which are essential for extracting correlated patterns in the swarm of data points in multidimensional space (for example, each relative gene expression profile with $\mathrm{k}$ time-points may be regarded as a k-dimensional vector).

\section{$2 \mathrm{SPC}$}

Currently, two clustering appoaches are very popular among biologists. One is average linkage, a hierarchical clustering method [5], with the Pearson correlation used as a similarity measure [6]. The other is self-organizing maps (SOMs) [7], whose most popular implementation for array data analysis is GENECLUSTER [8].

We present here clustering performed by SPC, a hierarchical clustering method recently introduced by Blatt et al [9]. It is based on an analogy to the physics of inhomogeneous ferromagnets. Full details of the algorithm [10] and the underlying philosophy [11] are given elsewhere ; here only a brief description is provided.

The input required for $\mathrm{SPC}$ is a distance matrix between the $N$ data points that are to be clustered. From such a distance matrix one constructs a graph, whose vertices are the data points and edges identify neighboring points. Two points $i$ and $j$ are called neighbors (and connected by an edge) if they satisfy the $K$-mutual-neighbor criterion, i.e. iff $j$ is one of the $K$ nearest points to $i$ and vice versus. With each edge we associate a weight $J_{i j}>0$, which decreases as the distance between points $i$ and $j$ increases.

Assignement of the datapoints to clusters is equivalent to partitioning this weighted graph. Cluster indices play the role of the states of Potts spins assigned to each vertex (i.e. to each original data point). Two neighboring spins

are interacting ferromagnetically with strength $J_{i j}$. This Potts ferromagnet is simulated at a sequence of temperatures $T$. The susceptibility and the correlation function for neighboring pairs of spins are measured. The pair correlation function serves to identify clusters: high correlation means that the two data points belong to the same cluster. 
The temperature $T$ controls the resolution at which clustering is performed; the algorithm finds typical clusters at all resolutions. At very low temperatures all points belong to a single cluster and as $T$ is increased, clusters break into smaller ones until at high enough temperatures each point forms its own cluster. The clusters found at all temperatures form a dendrogram. Blatt et al showed that the SPC algorithm is robust since the clusters are formed due to collective behavior of the system. The major splits can be easily identified by a peak in the susceptibility. For more details see [9-11].

\section{Yeast Cell Cycle and Microarray Data}

We applied SPC on a recently published data set [14] to determine whether it could automatically expose known clusters without using prior knowledge. Eisen et al [6] clustered the genes on the basis of data combined from 7 different experiments. We suspected that mixing the results of different experiments may introduce noise into the data associated with a single one. Therefore we chose to use only a single time course, that of gene expression as measured in a single process (cell division cycles following alpha-factor blockand-release [12]). Furthermore, we focused on genes that have characterized functions (2467 genes) for easier interpretation.

Genetic controls and regulation play a central role in determination of cell fate during development. They are also important for the timing of cell cycle events such as DNA replication, mitosis and cytokinesis. Yeast is a single cellular organism, which has become a favorite model in molecular biology due to the easiness of genetic and biochemical manipulation and the availability of the complete genome. Like all living cells, the yeast cell cycle consists of four phases: $\mathrm{G} 1 \rightarrow \mathrm{S} \rightarrow \mathrm{G} 2 \rightarrow \mathrm{M} \rightarrow \mathrm{G} 1 \ldots$, where $\mathrm{S}$ is the phase of DNA synthesis (replicating the genome); $\mathrm{M}$ stands for mitosis (division into two daughter cells), and the two gap phases are called G1 (preceding the S phase) and G2 (following the $\mathrm{S}$ phase). At least four different classes of cell cycle regulated genes exist in yeast [13]: G1 cyclins and DNA synthesis genes are expressed in late G1; histone genes in S; genes for transcription factors, cell cycle regulators and replication initiation proteins in G2; and genes needed for cell separation are expressed as cells enter G1. Early and late G1-specific transcription is mediated by the Swi5/Ace2 and Swi4/Swi6 classes of factors, respectively. Changes in the master cyclin/Cdc28 kinases are involved in all classes of regulation.

In the alpha-factor block-release experiments, MATa cells were first arrested in G1 by using alpha pheromone. Then the blocker was removed; from this point on the cell division cycle starts and the population progresses with significant cell cycle synchrony. RNA was extracted from the synchronized sample, as well as a control sample (asynchronous cultures of the same cells growning 
exponentially at the same temperature in the same medium).

Fluorescently labeled cDNA was synthesized using Cy3 ("green") for the control and Cy5 ("red") for the target. Mixtures of equal amounts of the two samples were taken at every $7 \mathrm{~min}$ and competitively hybridized to individual microarrays containing essentially all yeast genes. The ratio of red to green light intensity (proportional to the RNA concentrations) was measured by scanning laser microscopy (See [12] for experimental details). The actual data provided at the Stanford website [14] is the log ratios.

In the their analysis, Spellman et al. were focusing on identification of 800 cell cycle regulated genes (that may have periodic expression profiles). In our test of SPC, in addition to oscillatory genes we were also looking for any groups of genes with highly correlated expression patterns.

\section{SPC Analysis of Yeast Gene Expression Profiles}

We clustered the expression profiles of the 2467 yeast genes of known function over data taken at 18 time intervals (of $7 \mathrm{~min}$ ) during two cell division cycles, synchronised by alpha arrest and release. Denote by $E_{i j}$ the relative expression of gene $i$ at time interval $j$. Our data consist of 2467 points in an 18-dimensional space, normalised in the standard way:

$$
G_{i j}=\frac{E_{i j}-<E_{i}>}{\sigma_{i}} ; \quad<E_{i}>=\frac{1}{18} \sum_{j=1}^{18} E_{i j} ; \quad \sigma_{i}^{2}=\frac{1}{18} \sum_{j=1}^{18} E_{i j}^{2}-<E_{i}>^{2}
$$

We looked for clusters of genes with correlated expression profiles over the two division cycles. The SPC algorithm was used with $q=20$ component Potts spins, each interacting with those neighbors that satisfy the $K$-mutual neighbor criterion[10] with $K=10$. Euclidean distance between the normalized vectors was used as the distance between two genes. This distance is proportional to the Pearson correlation used by Eisen et. al..

At $T=0$ all datapoints form one cluster, which splits as the system is "heated". The resulting dendrogram of genes is presented in Fig. 1. Each node represents a cluster; only clusters of size larger than 6 genes are shown. The last such clusters of each branch, as well as non-terminal clusters that were selected for presentation and analysis (in a way described below) are shown as boxes. The circled boxes represent the clusters that are analysed below.

The position of every node along the horizontal axis is determined for the corresponding cluster according to a method introduced by Alon et al [4]; proximity of two clusters along this axis indicates that the corresponding temporal expression profiles are not very different. The vertical axis represents the 
resolution, controlled by the "temperature" $T \geq 0$. The vertical position of a node or box is determined by the value of $T$ at which it splits. A high vertical position indicates that the cluster is stable, i.e. contains a fair number of closely-spaced data points (genes with similar expression profiles).

In order to identify clusters of genes whose temporal variation is on the scale of the cell cycle, we calculated for each cluster a cycle score $S_{1}$, defined as follows. First, for each cluster $C$ (with $N_{C}$ genes) we calculate the average normalized expression level at all $j=1, \ldots, 18$ time intervals and the corresponding standard deviations $\sigma^{C}(j)$ :

$$
\bar{G}^{C}(j)=\frac{1}{N_{C}} \sum_{i \in C} G_{i j} \quad\left[\sigma^{C}(j)\right]^{2}=\frac{1}{N_{C}} \sum_{i \in C}\left(G_{i j}\right)^{2}-\left[\bar{G}^{C}(j)\right]^{2}
$$

Next, we evaluated the Fourier transform of the mean expression profiles $\bar{G}^{C}(j)$ for every gene cluster $C$. To suppress initial transients, the Fourier transform is performed only over $j=4, \ldots, 18$. Denote the absolute values of the Fourier coefficients by $A_{k}$; the ratio between low-frequency coefficients and the highfrequency ones was used as a figure of merit for the time scale of the variation. We observed that clusters that satisfy the condition

$$
S_{1}^{C}=\frac{\sum_{k=2}^{4} A_{k}}{\sum_{k=6}^{8} A_{k}}>2.15
$$

have the desired time dependence, and found 29 clusters (consisting of 167 genes) to have such scores. For many of these clusters, however, the temporal variation was very weak, i.e. of the same order as the standard deviations $\sigma^{C}(j)$ of the individual gene expressions of the cluster. We defined another score, $S_{2}^{C}$, for which we required

$$
S_{2}^{C}=\frac{1}{18} \sum_{j=1}^{18}\left[\frac{\bar{G}^{C}(j)}{\sigma^{C}(j)}\right]^{2}>5.6
$$

For clusters $C$ that satisfy this condition the "signal" significantly exceeds the noise. We select a cluster if its score exceeds 5.6, while its parent's score does not. Only 4 clusters, containing 86 genes, satisfy both conditions (1) and (2); these are numbered $1-4$ on Fig. 1. Seven additional relatively stable clusters which did not satisfy our two criteria, but are of interest, are also selected and circled on figure 1.

The corresponding time sequences are shown in Fig 2: $\bar{G}^{C}(j)$ is plotted for each cluster versus time $j$, with the error bars representing the standard deviations $\sigma^{C}(j)$. Clusters 1,2 and 4 clearly corresponds to the cell cycle. 


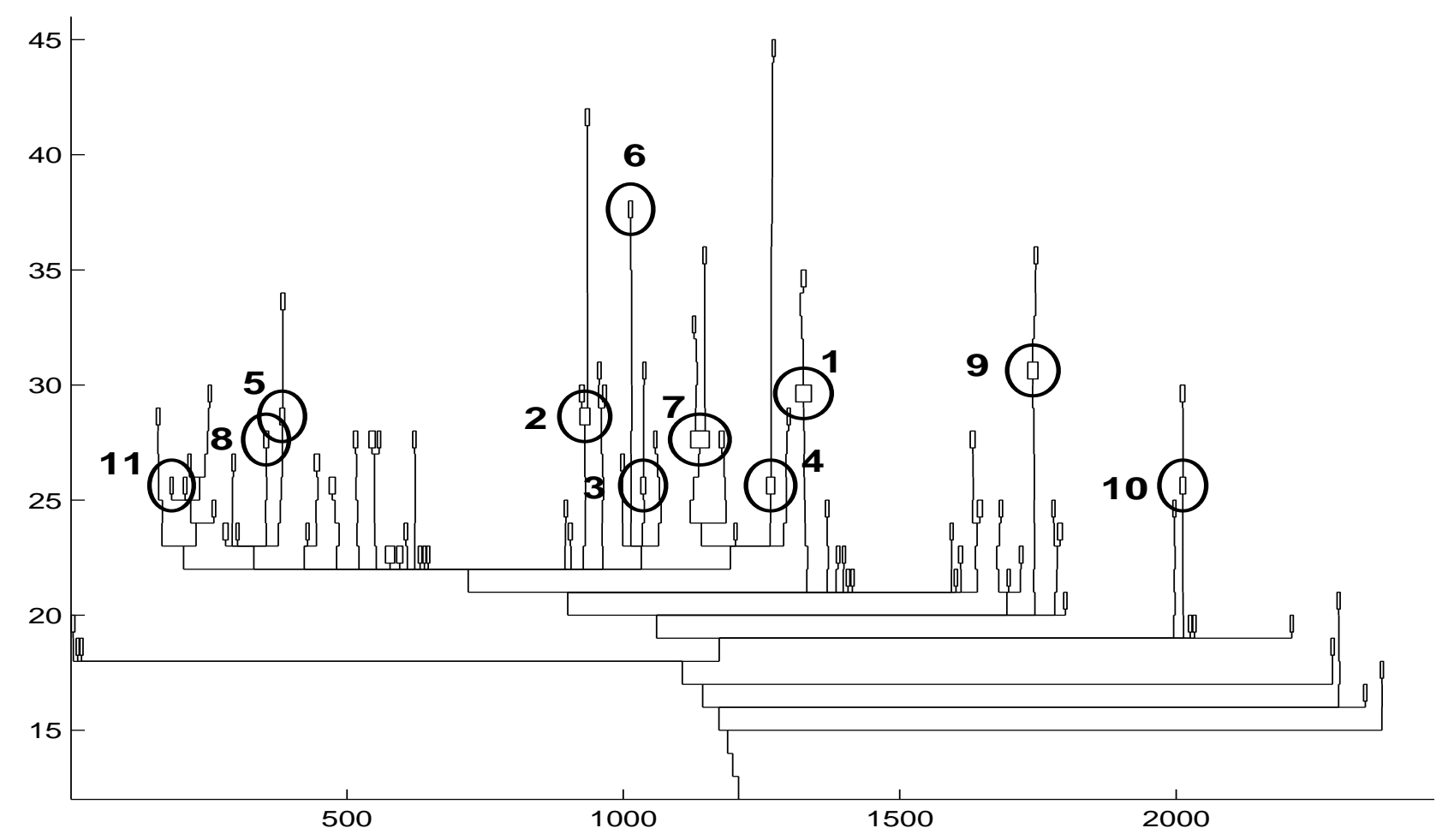

Fig. 1. Dendrogram of genes. Clusters 1 - 4 were selected according to our criteria, eq. (1 - 2). The other circled and numbered clusters are also interesting (see text).

\section{Details and Interpretation of gene clustering.}

The full lists of genes that constitute the 11 selected clusters are given in our website [15]. We present here a short analysis of our clusters. We use standard notation for bases: $\mathrm{R}$ stands for $\mathrm{A}$ or $\mathrm{G}, \mathrm{W}$ for $\mathrm{A}$ or $\mathrm{T}, \mathrm{K}$ for $\mathrm{G}$ or $\mathrm{T}, \mathrm{N}$ for any base.

Cluster \# 1: These are mostly Late G1 phase specific genes. They contain the major cell cycle regulators: Cln1,2, Clb5,6 and Swi4 as well as DNA replication and repair genes. One can easily detect MCB (ACGCGT) or SCB (CRCGAAA) sites in their promoters to which MBF (Swi6p+Mbp1p) and SBF (Swi6p+Swi4p) bind respectively [13].

Cluster \# 4: This cluster contains mostly S phase genes and is dominated by the histones. Histones are required for wrapping up nascent DNA into nucleosomes, their promoters are regulated by CCA (GCGAARYTNGRGAACR), NEG (CATTGNGCG) as well as SCB (CGCGAAA) [3].

Cluster \# 2: These are mostly G2/M phase genes. They contain the major cell cycle regulators: Clb1,2 and Swi5/Ace2. It is known that all genes co-regulated with Clb1,2 are mainly controlled by either Mcm1 at P-box 

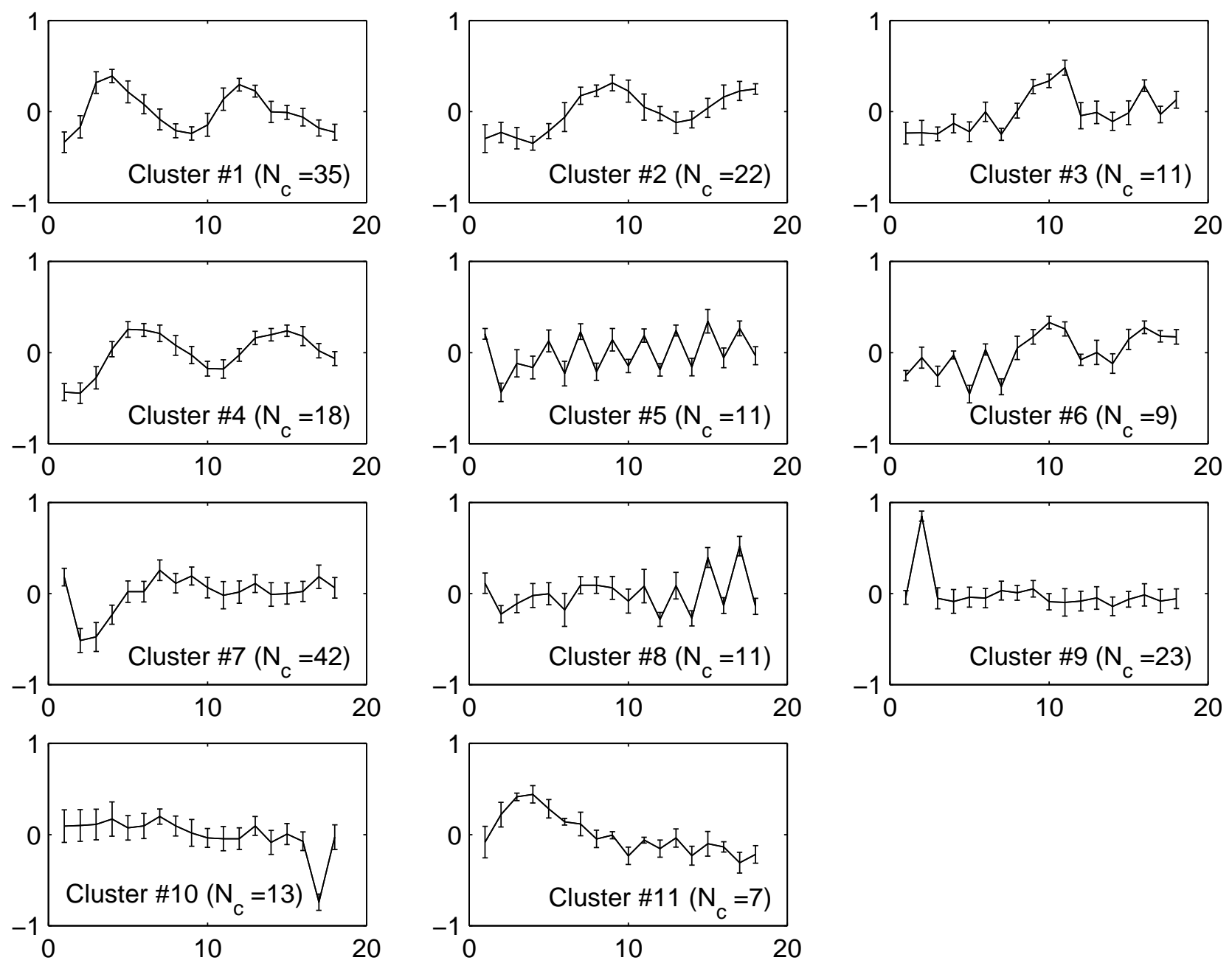

Fig. 2. Mean normalized expression of selected clusters, versus time, measured at intervals of 7 minutes. Error bars represent the standard deviations $\sigma^{C}(j) . N_{c}$ is the number of genes in each cluster. The clusters are numbered as in figure 1.

(TTWCCYAAWNNGGWAA) or by Mcm1+SFF through the composite site: (P-box)N2-4RTAAAYAA [12][3].

Clusters \# 5, \# 6 and \# 8: These are mostly ribosomal protein (RP) genes. The genome of Saccharomyces cerevisiae contains 137 genes coding for ribosomal proteins [16]. Since 59 genes are duplicated, the ribosomal gene family encodes 78 different proteins, 32 of the small and 46 of the large ribosomal subunit. They are co-regulated because they are sub-components of ribosome machinery for protein translation. All genes in cluster \#6 reside on chromosomes 2, 4 and 5, except rpl11b which resides on chromosome 9. All genes in clusters $\# 5$ and $\# 8$ (which are very close in the dendrogram of Figure 1) reside on chromosomes 8-16, except rps17b which resides on chromosome 4 . It is likely that the expression of these ribosomal genes is correlated to their chromosomal locations. It is interesting that the expression profiles appear to 
have pronounced oscillations (throughout in \#5, at early times in \#6 and late times in \#8). Like most of the RP genes, the ribosomal genes in the 3 clusters also contain multiple global Regulator Rap1p binding sites in their promoters within a preferred window of $15-26 \mathrm{bp}$ [17]. The transcription of most RP genes is activated by two Rap1p binding sites, 250 to 400 bp upstream from the initiation of transcription. Since Rap1p can be both an activator and a silencer, it is not known whether Rap1p is responsible for the oscillation. This oscillation could be a result of interplay between cell cycle and Rap1p activity which determines the mean half life of the RP mRNAs (5-7min, [18]). As fresh medium was added at $91 \mathrm{~min}$ during the alpha-factor experiments, the genes in \#6 and in \#8 may have different responses to the nutrient change.

Cluster \#7: This cluster has 42 genes that are largely not cell cycle regulated. These genes have diverse functions in general metabolism. When searching promoter regions for regulatory elements using gibbsDNA [20], a highly conserved motif GCGATGAGNT is shared by $90 \%$ of genes. This element seems to be novel, it has some similarity to Gcn4p site TGACTC and Yap1p site GCTGACTAATT [19]. When searching the yeast promoter database SCPD [21], we found that the BUF site in the HO gene promoter and the UASPHR site in the Rad50 promoter appear to contain the core motif GATGAG. Although we do not know if this element is functional or what might be the trans-factor, it is still very likely that it may contribute the co-regulation of this cluster of genes.

Cluster \#10: This cluster is characterized by a pronounced dip towards the end of the profile. They are not cell cycle regulated by and large, except Clb4 (a S/G2 cyclin ) and Rad54 (a G1 DNA repair gene). By searching promoter elements, we found a conserved motif RNNGCWGCNNC that is shared by a subset of the genes (Clb4, YNL252C, Rad54, Rpb10, Atp11 and Pex13). It partially matches a $\mathrm{PHO} 4$ binding motif (TCGGGCCACGTGCAGCGAT) in the promoter of Pho8. However, the PHO4 consensus, CACGTK, does not appear in the conserved motif of our cluster. Therefore we suspect that it is a novel motif which should be tested by experiments.

\section{Summary}

We used the SPC algorithm to cluster gene expression data for the yeast genome. We were able to identify groups of genes with highly correlated temporal variation. Three of the groups found clearly correspond to well known phases of the cell cycle; some of our observations of other clusters reveal features that have not been identified previously and may serve as the basis of future experimental investigations. 


\section{Acknowledgements}

Research of E. Domany was partially supported by the Germany-Israel Science

Foundation (GIF) and the Minerva foundation. Research of M. Q. Zhang was partially supported by NIH/NHGRI under the grant number HG01696.

\section{References}

[1] Lockhart DJ, Dong H, Byrne MC, et al. (1996) Nat. Biotech. 14, 1675-1680.

[2] De Risi J, Iyer V and Brown PO (1997) Science 278, 680-686.

[3] Zhang MQ (1999) Genome Res. 9, 681-688.

[4] Alon, U., Barkai, N., Notterman, D.A., Gish, K., Ybarra, S., Mack, D., AND Levine, A. J. (1999) Proc. Natl. Acad. Sci. 96, 6745-6750.

[5] Hartigan J (1975) Clustering Algorithms (Wiley, New York).

[6] Eisen M, Spellman PT, Brown PO and Botstein D (1998) Proc. Natl. Acad. Sci. USA 95, 14863-14868.

[7] Kohonen T (1997) Self-Organizing Maps (Springer, Berlin).

[8] Tamayo P, Slonim D, Mesirov J et al. (1999) Proc. Natl. Acad. Sci. USA 96, 2907-2912.

[9] Blatt, M., Wiseman, S., and Domany, E. 1996a. "Super-paramagnetic clustering of data", Physical Review Letters 76, 3251-3255.

[10] M. Blatt, S. Wiseman and E. Domany, Neural Computation 91805 (1997).

[11] E. Domany, Physica A 263, 158 (1999)

[12] Spellman PT, Sherlock G, Zhang MQ et al. (1998) Mol. Biol. Cell. 9, 3273-3297.

[13] Koch C and Nasmyth K (1994) Curr. Biol. 6, 451-459.

[14] The data can be obtained from http://cellcycle-www.standford.edu

[15] http://www.weizmann.ac.il/physics/complex/clustering/

[16] Mager WH, Planta RJ, Ballesta JG et al. (1997) Nucl. Acid. Res. 25, 4872-4875.

[17] Lascaris RF, Mager WH and Planta RJ (1999). Bioinformatics 15, 267-277.

[18] Li B, Nierras CR and Warner JR (1999). Mol Cell Biol 19, 5393-5404.

[19] Hinnebusch AG (1992). In The Molecular and Cellular Biology of the Yeast Sacchromyces: Gene Expression, Vol.2, pp319, Cold Spring Harbor Press, New York.

[20] Zhang MQ (1999a). Comput. Chem. 23, 233-250.

[21] Zhu J and Zhang MQ (1999). Bioinformatics 15, 607-611. 